\title{
Teratogenic Effect
}

National Cancer Institute

\section{Source}

National Cancer Institute. Teratogenic Effect. NCI Thesaurus. Code C50916.

The result of processes that negatively affect a developing fetus. 